\title{
Caminhos da Escola Austríaca: relação com ortodoxia, engajamento e produção de novo conhecimento
}

Paths of the Austrian School: its relationship with orthodoxy, engagement and production of new knowledge

Eduardo Angeli (1)

(1) Universidade Federal do Paraná

\section{Abstract}

The paper discusses the current situation of the Austrian School in three different matters. Firstly, the text argues that the Austrian School should not be considered orthodox, because it rejects one of the three neoclassical pillars, namely the emphasis on equilibrium states; in Austrianism, an analysis of market process is adopted. Secondly, the article shows that the Austrian school has, at least in part, sought greater engagement within the profession, instead of adopting a strategy of insulation. Finally, the text presents the reason why Austrian school should not be reduced to a closed chapter in the history of economic thought; it is argued that Austrianism has been trying to advance in contemporary theoretical debates and in the application of its unique approach to economic and social problems.

\section{Keywords}

austrian economics; history of economic thought; economic methodology.

JEL Codes B53; B49.

\section{Resumo}

$O$ artigo discute a situação corrente da Escola Austríaca em três aspectos. Em primeiro lugar, o texto argumenta que a Escola Austríaca não é ortodoxa, já que rejeita um dos três pilares neoclássicos, qual seja, a ênfase em estados de equilíbrio e o substitui pela análise do processo de mercado. Em segundo lugar, o artigo mostra que a Escola Austríaca tem, ao menos em parte, buscado um maior engajamento dentro da profissão, em vez de adotar a estratégia do isolamento. Por fim, o texto apresenta porque a Escola Austríaca não deve ser reduzida a um capitulo fechado e superado da história do pensamento econômico, tampouco como exclusivamente dedicada à HPE, na medida em que tem procurado avançar nos debates teóricos contemporâneos e na utilização de sua abordagem singular a problemas aplicados.

\section{Palavras-chave}

escola austríaca; história do pensamento econômico; metodologia da economia.

Códigos JEL B53; B49. 


\section{Introdução}

O presente texto procura contribuir para aprimorar o conhecimento dos economistas sobre a Escola Austríaca ao apresentar algumas características deste movimento intelectual. Para isso, o texto discute algumas imprecisões que ainda persistem a respeito da Escola Austríaca na comunidade dos economistas brasileiros.

Com os esclarecimentos sobre a Escola Austríaca e seu estado atual, por meio da crítica a algumas das concepções compartilhadas a seu respeito, o presente texto se adéqua à metodologia do pluralismo crítico (Caldwell, 1988), que defende a importância de se compreender melhor o movimento atual da disciplina Economia por meio da avaliação dos diferentes programas de pesquisa, de suas forças e fraquezas. Assim, "we will have a better understanding of what economic science is, and with luck that will lead to its improvement" (Caldwell, 1988, p. 240). Com isso, não se pretende que este seja um artigo apologético, mas de esclarecimento sobre a Escola Austríaca. Nesse sentido, representa uma contribuição a um esforço que tem sido feito por diferentes autores de melhorar a exposição sobre a metodologia, a singularidade e o estado atual do austrianismo (Aimar, 2009; Boettke, 2002a, 2011, 2015; Boettke et al., 2016; García-Brazales, 2002; Koppl, 2006; Rizzo, 2009; Stein e Storr, 2013).

Isso se mostra especialmente importante na medida em que as contribuições da Escola Austríaca permanecem pouco exploradas por parte dos economistas brasileiros. Uma busca, em abril de 2016, no sistema Scielo dá alguns indícios disso. Ao procurar no título ou resumo de artigos em revistas de Economia (Economia e Sociedade, Economia Aplicada, Estudos Econômicos, Nova Economia, Revista Brasileira de Economia, Revista de Economia Contemporânea, Revista de Economia Política e Revista de Economia e Sociologia Rural, cada uma delas coberta em diferentes períodos e números de volumes) surgem, para o termo "Hayek", apenas 15 artigos de 10 autores diferentes; já para o termo "Mises", apenas um artigo; para "Escola Austríaca", nenhum artigo.

Antes de dar continuidade a este texto, cabe observar que, especialmente nas seções 3 e 4 , quando se fala do austrianismo contemporâneo, trata-se desta corrente do pensamento nos EUA (para onde ela migrou na primeira metade do século XX e onde ocorreu seu ressurgimento em meados dos anos 1970 - ver Vaughn, 1994). Deve-se ressaltar, contudo, que importan- 
tes contribuições de economistas Austríacos ou, de modo mais geral, que se valem de modo relativamente amplo de autores Austríacos, têm sido feitas fora dos EUA, especialmente na Europa. Nesse caso, merecem destaque trabalhos de autores franceses como Pierre Garrouste, Agnès Festré e Thierry Aimar, e de britânicos como Paul Lewis e Mark Pennington. Contudo, no presente trabalho está subjacente que é nos EUA que o austrianismo tem maior número de adeptos que se identificam como participantes da Escola, bem como maior grau de organização acadêmica, com ao menos um programa de doutorado com um campo de estudo em Economia Austríaca (Universidade George Mason), ao menos duas revistas acadêmicas listadas em artigos como Combes e Linnemer (2010) e Lee et al. (2010) (Review of Austrian Economics e Quarterly Journal of Austrian Economics) e duas organizações (Society for the Development of Austrian Economics e Mises Institute).

Diante disso, o presente artigo procura discutir, em cada uma das três seções seguintes, três supostas características da Escola Austríaca que podem distorcer a compreensão que estudantes e economistas desenvolvem a respeito de tal Escola: (i) a Escola Austríaca é ortodoxa; (ii) a Escola Austríaca é isolacionista; (iii) a Escola Austríaca não tem produzido conhecimento novo e relevante. $O$ texto é concluído com as considerações finais.

\section{A Escola Austríaca é ortodoxa?}

O conceito de ortodoxia não é consensual entre os economistas. Para os propósitos deste texto, é seguida a definição de Dequech (2007), que trata de três categorias: ortodoxia, heterodoxia e mainstream. Em particular com relação ao termo ortodoxia, ele a identifica atualmente com a Escola Neoclássica, que é assentada sobre três pilares: destaque para racionalidade (entendida como maximização de utilidade), ênfase sobre estado(s) de equilíbrio e negligência de tipos fortes de incerteza. Pode-se argumentar que a Escola Austríaca rejeita estes três pontos e, portanto, não pode ser considerada ortodoxa. Contudo, por limitação de espaço, destaque será dado para a substituição da ênfase em equilíbrio pela abordagem do processo de mercado - ao rejeitar um dos três pilares, mostra-se que há sim uma importante separação entre Economia Ortodoxa e Economia Austríaca. ${ }^{1}$

1 Dequech (2007) usa um conceito sociológico (e não intelectual, como no caso da Orotodoxia) para definir Mainstream: a Economia Mainstream é aquilo "taught in the most prestigious 
Posto isto, a presente seção apresenta sumariamente como alguns dos expoentes do austrianismo trataram o processo de mercado, de modo a marcar a diferença entre tal abordagem e a da ortodoxia. Adicionalmente, mostra que na própria definição que economistas Austríacos dão para a Escola, fica evidente a importância atribuída ao processo de mercado, em oposição à escolha pelo estudo de estados de equilíbrio.

A rejeição da opção pelo estudo de estados de equilíbrio caracteriza a separação da Escola Austríaca em relação à Economia ortodoxa. Mas do que se trata o processo de mercado? Segundo Mises:

The market is not a place, a thing, or a collective entity. The market is a process, actuated by the interplay of the actions of the various individuals cooperating under the division of labor. The forces determining the - continually changing - state of the market are the value judgments of these individuals and their actions as directed by these value judgments. (...) The market process is entirely a resultant of human actions. Every market phenomenon can be traced back to definite choices of the members of the market society. The market process is the adjustment of the individual actions of the various members of the market society to the requirements of mutual cooperation. (Mises, 2007, p. 257-258).

O processo de mercado é resultado da ação humana. Ao estudar a ação humana, a Economia (ou, de modo mais geral, a praxeologia proposta por Mises) apresenta-se de forma fundamentalmente separada das ciências naturais. Nas palavras do autor:

[T] here is in the universe something for the description and analysis of which the natural sciences cannot contribute anything. There are events beyond the range of those events that the procedures of the natural sciences are fit to observe and to describe. There is human action. (Mises, 2006, p. xi).

Mises (2007, cap. 14) afirma que o método da Economia (e da praxeologia) é o das construções imaginárias, em que o economista abstrai características que envolvem a ação humana para poder avaliar as consequências dessa ausência em um plano abstrato, bem como para compreender os efeitos de sua existência. Para isso, o economista se vale do apriorismo, a dedução a partir de premissas evidentemente verdadeiras, em última instância, o axioma da ação humana. Por serem erigidas a partir de abstrações da realidade, e por se valerem da dedução apriorística, não faz sentido, para Mises, tentar avaliar empiricamente as construções imaginárias. Ao contrário, a utilidade delas é devida precisamente às dificuldades próprias important research foundations, and wins the most prestigious awards". (Dequech, 2007, p. 281). 
da praxeologia, em sua peculiaridade frente às ciências naturais, de se utilizar a realidade empírica:

Praxeology cannot, like the natural sciences, base its teachings upon laboratory experiments and sensory perception of external objects. It had to develop methods entirely different from those of physics and biology. It would be a serious blunder to look for analogies to the imaginary constructions in the field of the natural sciences. The imaginary constructions of praxeology can never be confronted with any experience of things external and can never be appraised from the point of view of such experience. Their function is to serve man in a scrutiny which cannot rely upon his senses. In confronting the imaginary constructions with reality we cannot raise the question of whether they correspond to experience and depict adequately the empirical data. (Mises, 2007, p. 236-237).

Entre as construções imaginárias de Mises (e, portanto, não descrições da realidade) está o final state of rest, compatível com uma situação em que não há pressões para alteração de preços e que, para o autor, não pode ser atingida. Segundo Mises, "the market at every instant is moving toward a final state of rest. Every later new instant can create new facts altering this final state of rest. But the market is always disquieted by a striving after a final state of rest" (Mises, 2007, p. 245).

Tal visão de Mises foi desenvolvida posteriormente em especial por Kirzner (e.g. 2013), que utiliza a explicação do processo de mercado posto em movimento pela ação do empresário dotado do estado de alerta. Tal atividade empresarial é o que, ao proporcionar a descoberta de oportunidades de ganho e explorá-las, promove o movimento do sistema econômico rumo a um estado mais próximo do equilíbrio, que nunca é alcançado. ${ }^{2}$

Hayek (e.g. 1937; 1945; 1979, cap. 3), por sua vez, destacou o caráter subjetivo, incompleto e difuso do conhecimento possuído pelas pessoas. Para Hayek, este é um fato fundamental com o qual as ciências sociais deveriam lidar. Na perspectiva de Hayek, é razoável supor que o indivíduo esteja em equilíbrio, no sentido de que seus planos apresentem coerência e compatibilidade com o conhecimento que ele possui. Contudo, a passagem do equilíbrio individual para o equilíbrio geral (no sentido de envolver todos os participantes do mercado) não é trivial, na medida em que requer compatibilidade entre os planos dos inúmeros participantes do mercado, em particular com relação ao comportamento esperado dos demais indivíduos. Diante disso, cumpriria aos economistas explicar a passagem do

2 Sobre o debate entre Kirzner e Lachmann sobre uma tendência ao equilíbrio, ver Barbieri (2008). 
equilíbrio individual ao geral por meio da maior compatibilização do conhecimento e dos planos. O problema da maior parte dos economistas é que a escolha metodológica deles é estudar estados de equilíbrio, admitindo conhecimento perfeito. Supõem, assim, o que deveriam explicar. Por isso a necessidade de estudo do processo de mercado, onde acontece a revisão de planos com a descoberta, transmissão e difusão de conhecimento.

Assim, pode-se argumentar que o problema central, para a Economia Austríaca, é o problema do conhecimento, e que isso se vincula à opção não de se estudar estados de equilíbrio, mas o processo de mercado por meio do qual ocorre a coordenação de planos. Tal fato fica explícito na própria definição de Escola Austríaca que, como veremos abaixo, dá destaque ao processo de mercado como tema central, segundo diferentes autores.

Boettke (1994) fala que, a despeito da heterogeneidade existente dentro do austrianismo, existem ao menos dois objetivos e três princípios metodológicos que conferem ao austrianismo um grau de coesão que justifica a utilização do termo "Escola". Os objetivos perseguidos por um autor austríaco seriam (i) apresentar os eventos econômicos em termos da ação humana direcionada a um propósito (purposive human action) e (ii) relatar as consequências não intencionais dessa ação humana. Para atingir estes dois objetivos, os três postulados metodológicos seriam: (i) individualismo metodológico; (ii) subjetivismo metodológico; e (iii) foco em processos ao invés de estados de equilíbrio.

Já Rizzo (2009, 2013), mais voltado aos desenvolvimentos recentes feitos pelo austrianismo, cita oito temas que caracterizam a identidade Austríaca. Nas suas palavras:

(1) the subjective, yet socially embedded, quality of human decision making; (2) the individual's perception of the passage of time ('real time'); (3) the radical uncertainty of expectations; (4) the decentralization of explicit and tacit knowledge in society; (5) the dynamic market processes generated by individual action, especially entrepreneurship; (6) the function of the price system in transmitting knowledge; (7) the supplementary role of cultural norms and other cultural products ('institutions') in conveying knowledge; and (8) the spontaneous - that is, not centrally directed - evolution of social institutions. (Rizzo, 2013, p. 50-51).

Kirzner (1987), por sua vez, mostra que, no começo da década de 1930, a Escola Austríaca era reconhecida como um capítulo importante, mas já encerrado, da história do pensamento econômico. Em parte isso se devia a que muitos dos Austríacos entendiam que o austrianismo havia sido ab- 
sorvido pela corrente principal da profissão, deixando de existir razão que justificasse uma escola autônoma, seja pela vitória da posição defendida por Menger no Methodenstreit a respeito da possibilidade de teorização em Economia contra a crítica historicista, seja porque muitas das contribuições Austríacas à teoria da econômica haviam sido incorporadas pelos economistas de fora da Áustria (Kirzner, 1987).

Tal visão teria surgido com a geração de Böhm-Bawerk e Wieser, que se enxergavam não como uma alternativa radical à abordagem mainstream, mas como colaboradores dela - ao contrário de Menger, que procurou guardar distância entre sua abordagem e as de Walras e Jevons (Boettke, 1994).

Nesse espírito, Machlup levantou seis características da Escola Austríaca antes da segunda guerra mundial:

\begin{abstract}
(a) methodological individualism (not to be confused with political or ideological individualism, but refering to the claim that economic phenomena are to be explained by going back to the actions of individuals); (b) methodological subjectivism (recognizing that the actions of individuals are to be understood only by reference to the knowledge, beliefs, perception and expectations of these individuals); (c) marginalism (emphasizing the significance of prospective changes in relevant magnitudes confronting the decision maker); (d) the influence of utility (and diminishing marginal utility) on demand and thus on market prices; (e) opportunity costs (recognizing that the costs that affect decisions are those that express the most important of the alternative opportunities being sacrificed in employing productive services for one purpose rather than for the sacrificed alternatives); ( $f$ ) the time structure of consumption and production (expressing time preferences and the productivity of 'roundaboutness'). (Kirzner, 1987, p. 64-65).
\end{abstract}

De acordo com Kirzner, tal lista de Machlup seria uma descrição não só do austrianismo pré-Guerra, mas da maior parte da microeconomia moderna - o que daria sustentação à ideia de absorção do austrianismo pela corrente principal da profissão. Contudo, Kirzner argumenta que avanços realizados posteriormente fizeram com que a lista de Machlup ficasse incompleta - algo que dificilmente poderia ter sido previsto, a despeito de estes desenvolvimentos mais recentes terem estado latentes nas contribuições Austríacas anteriores. Os dois elementos que afloraram após os anos 1930 seriam os seguintes: " $(g)$ markets (and competition) as processes of learning and discovery; (h) the individual decision as an act of choice in an essentially uncertain context (where the identification of the relevant alternatives is part of the decision itself)" (Kirzner, 1987, p. 66).

Kirzner (1987) argumenta que tais desenvolvimentos feitos pela Escola Austríaca a partir da década de 1930, com as contribuições de Mises e Ha- 
yek ao debate do cálculo econômico socialista, apontam para importantes diferenças entre o austrianismo e a corrente principal da profissão. Ambas as características distintivas estão assentadas sobre a rejeição da análise de equilíbrio tal qual feita pela Economia convencional (e pelos defensores do socialismo naquele debate), o que levou Mises e Hayek a exporem a oposição Austríaca a este tipo de análise em favor da ênfase no tripé "processo, aprendizado e descoberta". Em texto anterior, Kirzner dá especial ênfase a este aspecto: "[a] characteristic feature of the Austrian approach to economic theory is its emphasis on the market as a process, rather than as a configuration of prices, qualities, and quantities that are consistent with each other in that they produce a market equilibrium situation" (Kirzner, 1974, p. 3, grifo retirado). ${ }^{3}$

Na mesma linha, segundo Boettke (1994), mesmo durante os anos 1930, em que vigorava a concepção de que o austrianismo havia se fundido ao restante da teoria econômica, permaneciam peculiaridades relevantes dos Austríacos: o fato de não se remeter à teorização matematizada, de utilizar argumentos filosóficos, de destacar o caráter dinâmico do mercado e de lidar com questões sociais e políticas que ultrapassavam o objeto usual do economista (as trocas e a produção). Dessa maneira, "[t]he Austrians were quintessential members of the mainstream economic thought, but their methodological position and analytical approach in monetary theory, capital theory and even basic price theory forced them into increasing confrontations with the mainstream of English language economics" (Boettke, 1994, p. 2).

Já de acordo com Lachmann (1978), três características marcariam a singularidade do austrianismo face outras correntes de pensamento econômico: (i) o radical subjetivismo que inclui não só preferências, mas também expectativas; (ii) a inclusão do tempo como variável relevante ao estudo da economia, com particular atenção às alterações no conhecimento disponível; e (iii) a desconfiança com relação à utilização de categorias que

3 De fato, pode-se argumentar que, ironicamente, foi o uso das ferramentas usuais dos economistas por parte dos defensores do socialismo, como Lange, durante o debate do cálculo econômico socialista, que levaram Mises e Hayek a reformularem a posição Austríaca, tornando explícita sua separação da corrente principal da profissão e colocando-a em oposição ao que viria a ser dominante em economia nas décadas seguintes - vale dizer, o processo de exclusão da Escola Austríaca do que então se constituía como Mainstream Economics. Ver, a respeito, Barbieri (2013) e Boettke et al. (2016). Vaughn (1994) também narra esta história, além de mostrar como a Escola Austríaca - após ter passado por uma profunda crise provocada por sua postura contrária aos desenvolvimentos contemporâneos da Economia (curiosamente, ao mesmo tempo em que Mises e Hayek davam algumas de suas principais contribuições) e de ter "migrado" para os EUA - viveu uma espécie de ressurgimento a partir da década de 1970. 
não sejam expressões do que existe nas mentes do homo agens misesiano, em particular a utilização de agregados macroeconômicos e o estudo de suas relações.

No presente texto destaque especial é dado à linha Mises-Hayek-Kirzner, além de Lachmann. Isso não significa, contudo, que não existam tensões entre os Austríacos até mesmo com relação à definição do que é Austríaco e ao nome da Escola.

Rothbard (1992) argumenta que o austrianismo deveria ser definido de uma maneira mais estrita em relação ao apresentado no presente texto. Segundo ele, a definição do que pode ser considerado como Economia Austríaca usualmente é tão ampla que chega a incluir propostas por vezes opostas e conflitantes. Segundo ele:

It has unfortunately become habitual in summing up Austrian economics, or the Austrian paradigm, to present it as an unconnected grab-bag of separate principles, a laundry-list of various separate traits: In particular, "subjectivism"; "market process" or disequilibrium processes as against equilibrium or end-states; market coordination of plans; methodological individualism; stress on the "unintended consequences" rather than the intended consequences of human action; and writing in "literary" style or ordinary language rather than in formal mathematics. (...) [T] his emphasis on the unconnected laundry-list leads almost inevitably into gross error, for it leads to a one-sided overvaluation and therefore mis-emphasis on such particular traits as "subjectivism," "market process," or unintended consequences, thereby unfortunately denigrating such other crucial elements of Austrianism as objective reality and its laws, the end-state or equilibrium goals implicit in all human action, and the exercise of reason and therefore the intended consequences of such action. (Rothbard, 1992, p. 170).

Rothbard, assim, sugere uma oposição entre a abordagem de Lachmann e possivelmente Hayek (subjetivismo, consequências não-intencionais) e a sua própria, que seria herdeira da de Mises (ação humana e consequências intencionais, individualismo metodológico). Em seu texto, Rothbard procura rebater cada um dos elementos usualmente listados como características do austrianismo pelo que ele qualifica como "non-Misesian Austrian (Hayekian and others)" (Rothbard, 1992, p. 171), como o subjetivismo defendido por "Lachmannians and other pseudo-Austrians" (Rothbard, 1992, p. 172).

De forma semelhante, Klein (2008) argumenta que a ênfase em elementos como subjetivismo, processo de mercado, incerteza, consequências não-intencionais, evolução institucional etc., normalmente associados a autores como Hayek, Lachmann e Kirzner, desloca o foco do que realmen- 
te seria central ao austrianismo, o que ele chama de mundane economics: "price theory, capital theory, monetary theory, business-cycle theory, and the theory of interventionsim" (Klein, 2008, p. 167), vale dizer, temas que poderiam provocar confusão entre o austrianismo e a Economia mainstream, mas que são tratados de uma perspectiva singular, que passa pela abordagem causal-realista que seria o legado de Menger (Klein, 2006).

Um debate ocorrido recentemente tanto em revistas quanto em blogs passou, entre outras questões, pelo uso do termo "Escola Austríaca". Ele envolveu a sugestão, por parte de autores como Block (2012), de que um grupo de Austríacos deixasse de se identificar com esta Escola e adotasse outro nome, assumindo seu caráter supostamente "cismático". Segundo Block (2012), a praxeologia tal qual usada por Mises e Rothbard seria o caso paradigmático do austrianismo, de modo que críticas a ela representariam um desvio da tradição. A razão imediata da crítica de Block (2012) foi a publicação de Prychitko (2002). Block cita órgãos vinculados ao grupo ao qual Prychitko pertence, como a Society for the Development of Austrian Economics (SDAE) e a Review of Austrian Economics (RAE). O blog de Boettke, Prychitko e outros, de fato, havia tido o nome trocado já em $2010 \mathrm{em}$ virtude das disputas entre os grupos, deixando de ser o Austrian Economists para ser o Coordination Problem. ${ }^{4}$

Segundo Egger (1978), a singularidade Austríaca frente ao que ele chama de ortodoxia neoclássica está, entre outras coisas, na adoção da hipótese de informação imperfeita, no papel da ação empresarial no processo de mercado e na inclusão do tempo como variável relevante na explicação econômica. Boettke (2002a) e Barbieri (2006) falam da distinção entre o problema da informação (incorporado ao mainstream) e o problema do conhecimento de Hayek, ao qual Egger faz referência. A propósito, o movimento de incorporação e conversão do problema do conhecimento de Hayek na Economia da informação neoclássica pode ser visto como mais um capítulo da "cheia do mainstream" de que fala Possas (1997). Como nota Rizzo:

If we take time seriously, it is hard to imagine Austrian economics as merely "a supplement to neoclassical economics." (...) Some neoclassical economists may be able to make improvements in their analyses by formalizing (and thus transforming) the insights to Menger, Hayek or others. But this is not the essence of the Austrian contribution to knowledge about the social world. Austrians ask different kinds of 
questions and provide different kinds of answers. This is not to say that they may not sometimes ask the same or similar questions or that their vocabulary might not be at least superficially similar to that of the neoclassical mainstream. It is to say, however, that Austrian economics is essentially a different enterprise from neoclassical social physics. (Rizzo, 1996, p. xiii).

Das discussões acima mencionadas sobre o que é a Escola Austríaca, merece destaque a concepção de que o austrianismo está intimamente associado ao conceito de processo de mercado. ${ }^{5}$ A propósito, o destaque que a substituição da análise de equilíbrio pela de processo tem na definição do que é a Escola Austríaca é tão grande que, de acordo com Vaughn (2014), em seu início o grupo austríaco da George Mason University (GMU), hoje um dos redutos Austríacos no mundo, chamava-se Center for the Study of Market Process, e não "programa de Economia Austríaca". James Buchanan, antes de entrar para o corpo docente da GMU no início da década de 1980, teria apreciado a escolha do nome: "[h]e felt it was important to focus on a research question rather than on a particular ancestry" (Vaughn, 2014, p. 162, n. 6). O grupo austríaco existente na New York University, liderado por Mario Rizzo, chama-se Colloquium on Market Institutions \& Economic Processes.

Em suma, clássicos do austrianismo como Mises, Hayek e Kirzner, distanciam-se do uso convencional do equilíbrio. Isso, alinhado ao conhecimento de que, de forma geral, os Austríacos se valem da noção de extended present de que fala Boulding (1971), no relacionamento com autores do passado, permite concluir que existe uma separação teórico-metodológica importante entre os Austríacos e Economia Ortodoxa. ${ }^{6}$ Cumpre destacar que, em oposição à Mainstream Economics, Boettke (2007) fala de uma Mainline Economics, uma categoria intelectual que procura englobar autores e escolas que, ao longo da história do pensamento econômico, buscaram e buscam explicar como se dá o processo (espontâneo) de harmonização de

5 Para uma descrição mais esquemática da Escola Austríaca, ver o sumário proposto em Mair e Miller (orgs.)(1991, p. 68-70).

6 O Principle of the Extended Present de que fala Boulding (1971) diz respeito, em suma, à percepção de que nas mais diferentes áreas do conhecimento humano os debates não acontecem em um ponto específico do tempo, mas ao longo do tempo. Assim, textos são publicados utilizando ou comentando outros textos publicados no passado, de modo que um autor, mesmo que já esteja morto, de certa forma pertence ao presente na medida em que suas produções ainda são objeto de discussão. A questão, portanto, diz respeito não ao uso de textos e autores do passado, mas de quão longo o extended present é em cada disciplina. Na visão da maior parte dos economistas, como argumentam Boettke et al. (2014), ele seria bastante estreito, o que estaria relacionado com a progressiva perda de prestígio da HPE. Entre os Austríacos, por outro lado, ele seria bem mais amplo. 
interesses opostos e de cooperação anônima por meio do mecanismo de mercado. Nesta categoria poderiam ser enquadrados autores como Smith, Menger, Friedman, Mises, Buchanan e Hayek, por exemplo.?

\section{A Escola Austríaca é isolacionista?}

Segundo Aimar (2009), um dos elementos que tem dificultado o aumento da influência do austrianismo sobre a comunidade dos economistas é o uso por vezes belicista que se faz dele com vistas a servir de suporte para propósitos políticos. Tal politização provoca, de um lado, a criação de uma caricatura do austrianismo por parte, em geral, dos opositores do liberalismo, posição associada à Escola, ${ }^{8}$ e, de outro, a ampliação do preconceito de que os Austríacos não podem contribuir com os debates contemporâneos em teoria econômica. Em outras palavras, o fato de que ao menos parte da teoria Austríaca acaba sendo usada para "partisan ends" (Aimar, 2009, p. 201), ou seja, como apêndice ideológico para certas posições políticas, a tornaria incapaz de dialogar e contribuir para os debates relevantes contemporâneos em teoria econômica.

A presente seção procura discutir se a caracterização do austrianismo como uma corrente belicista, um grupo que se preocupa mais em atacar os que não aderem aos seus próprios textos clássicos, ao invés de contribuir de forma engajada, é correta. $\bigcirc$ argumento é que tal caracterização não pode ser generalizada para toda a Escola.

Kirzner, ao discutir os significados associados à Escola Austríaca na segunda metade dos anos 1980, afirma que para alguns a Escola Austríaca seria mais facilmente identificada com o libertarianismo do que com alguma proposta metodológica singular, de modo que "[f]or those observers, to be an Austrian economist in the 1980's is simply to be in favour of free markets" (Kirzner, 1987, p. 67).

7 Para uma discussão sobre a relação entre a Mainline Economics e as categorias Ortodoxia, Heterodoxia e Mainstream Economics, tais como propostas por Dequech (2007), ver Angeli (2014).

8 Boettke (1995) estuda a relação entre liberalismo e austrianismo. Interessante notar que um dos principais estudantes e depois colegas de Menger, Wieser (de quem Mises e Hayek foram alunos) admitia um grau de participação do Estado maior do que usualmente é associado ao austrianismo: "[a]mong the founders of the school, Wieser was in fact explicit in endorsing the interventionist conclusions of the German Historical School" (Kirzner, 1987, p. 68). 
Segundo Kirzner, esta identificação é devida à firme defesa que Mises fazia da causa liberal, a ponto de isso parecer ser o núcleo da posição Austríaca, o que teria sido aprofundado por um de seus principais seguidores, Murray Rothbard. No entanto, ainda segundo Kirzner (1987), esta visão não é precisa, tanto que Mises e Hayek defendiam que suas contribuições à teoria econômica prezavam a neutralidade, e que a partir disso é que questionavam a possibilidade do socialismo, de modo que "[b]oth writers would see their free market stance at the policy level as related to, but not central to, their Austrianism" (Kirzner, 1987, p. 68).

Na mesma linha, mas falando de Mises e Rothbard, Block (2012) explica que ambos os autores reconheciam a distinção entre postulados normativos, de um lado, e postulados positivos, de outro, ${ }^{9}$ de modo que defendiam a neutralidade de suas proposições em Economia e que a "political economic philosophy" (Block, p. 156) que adotavam não possuía relação direta com a abordagem praxeológica que defendiam. Segundo sua visão, "[t]here is in fact a gigantic logical divide between Austrian economics, on the one hand, and libertarian political philosophy, on the other" (Block, 2012, p. 159, grifo no original), em virtude da distinção entre ciência positiva (a Economia Austríaca) e disciplina normativa (o libertarianismo) - o que não impede que os tipos de perguntas levantados à Economia Austríaca e ao libertarianismo tenham alguma relação. ${ }^{10}$

9 Quais sejam, a defesa do "limited government libertarianism for Mises and anarcho-capitalism for Rothbard". (Block, 2012, p. 156).

10 "Austrian economics, as in the case of all other schools of thought in the "dismal science," is concerned with issues such as: What causes what? How do we explain and understand economic reality? How are prices determined? Why do we have depressions? What are the effects of tariffs? Libertarianism, in sharp contrast, addresses itself to an entirely different set of issues. It asks: Under what conditions is the use of force justified? Should abortion be allowed? Would voluntary slave contracts be legitimate in a free society? Is the death penalty justified? Is the minimum-wage law legitimate? What immigration policy is compatible with libertarianism? Now, of course, the two sets of questions are not entirely unrelated. For example, whether or not the minimum-wage law is compatible with justice in some measure depends upon what, precisely, are its effects" (Block, 2012, p. 159). Por outro lado, Kirzner, apesar de julgar que "Rothbard was unquestionably a genious" (Kirzner, 1997), afirma que teve pontos de desacordo com Rothbard "in matters of style and in matters of substance" (Kirzner, 1997). Segundo Kirzner, "[s]ome of his [Rothbard's] impact was deepened, and some of it qualified, by his ideological work in libertarian political theory" (Kirzner, 1997). Em um excerto de entrevista disponível em https://youtu.be/Z_cGdqUfN0c (acessado em 10/04/2016), Kirzner vai além, ao afirmar que a contribuição de Rothbard à Economia Austríaca é borrada porque, na perspectiva de Rothbard, não se precisaria separar ciência e julgamentos de valor, de modo que a Economia e o libertarianismo de Rothbard estariam intrinsecamente conectados um ao outro. Isso, na interpretação de Kirzner, atrapalharia a imagem e o impacto do austrianismo, ainda que possa ser importante na atração, para a Economia Austríaca, de jovens interessados em libertarianismo. Em sua própria perspectiva, a Economia Austríaca não deve se deixar confundir 
Um dos principais expoentes do Austrianismo contemporâneo, Boettke (2011) defende que os economistas Austríacos devem admitir que seu objetivo, enquanto economistas acadêmicos, deve ser publicar trabalhos relevantes e inovadores nas melhores revistas e que venham a ser muito citados, e lecionar Economia para os melhores alunos. Assim, segundo ele, é preciso que se diga claramente que um economista austríaco almeja (ou pelo menos deveria almejar) o seguinte:

[T]o publish in the American Economic Review (AER) not the Review of Austrian Economics (RAE), or the Quarterly Journal of Economics not the Quarterly Journal of Austrian Economics; to hold positions at Harvard University, the University of Chicago, Princeton University, Stanford University, or MIT rather than GMU, West Virginia University, Clemson University, Florida State University or Suffolk University (...). It is what it is, and to deny reality is to make a serious mistake. (Boettke, 2011, p. 20).

Adicionalmente, ele explicita:

Just to be clear, what we are trying to pull off is no less of an accomplishment than what happened with the Keynesian revolution mid 20th century. We want to place an Austrian inspired economist in every university and college in the US within the next 30 years, and clusters of centers for research and education in 20 or more schools, with half of those being in graduate programs and at least 1 or 2 in elite $\mathrm{PhD}$ programs. (Boettke, 2011, p. 21).

A posição de Boettke chama a atenção por refletir uma postura a favor do engajamento do Austrianismo dentro do amplo campo de trabalho dos economistas acadêmicos, em particular com relação à "métrica" do sucesso de um economista. Boettke rejeita o isolacionismo e o "belicismo" que parecem marcar ainda boa parte do austrianismo. Ele lamenta que tal engajamento acadêmico ao espectro maior da profissão ainda não seja amplamente difundido entre os Austríacos.

A estratégia proposta por Boettke é que o austrianismo não se resuma a guetos que vivam a atacar os de fora. Para ele, o economista austríaco deve romper os muros da própria Escola e utilizar Hayek e Mises como equipamento para discutir com o restante da profissão temas relevantes e se abrir para propostas de aproximações teóricas e complementação na compreensão do mundo. Em seus conselhos para estudantes Austríacos, Boettke diz o seguinte:

com o libertarianismo, mas deve ser tratada como uma "purely scientific intellectual endeavor", o que não estaria de acordo com o pensamento e a ação de Rothbard. 
Academic life is too short and your professional colleagues are too interesting to emphasize differences, rather than commonalities. Constantly seek to find common ground from which to work with the purpose of tackling relevant problems. (...) Our task is one of engagement with our colleagues and our students, not isolation and insulation. (Boettke, 2011, p. 22).

Ainda em sua perspectiva, outro ponto que os Austríacos devem imitar de Mises e Hayek é a postura com relação aos trabalhos e autores de fora da própria tradição Austríaca. Segundo seu relato, ambos os expoentes do austrianismo rejeitavam rótulos, ainda que abraçassem sua herança Austríaca. Tal postura deve ser resultado da visão de que o que importa não são rótulos que as pessoas ostentam em termos de orientação e fidelidade teórica, mas o tipo de argumento proposto e as posições tomadas, de modo que, mais do que tentar beber de fontes que se autodenominam Austríacas, o austrianismo deve se abrir para oportunidades de intercâmbio e ganho intelectual com o restante da profissão.

Assim, destaque deve ser dado a essa mudança de postura que tem caracterizado ao menos parte do grupo de economistas Austríacos. Se parte do austrianismo ainda toma atitude "belicistas", isolacionistas, e é, de certa forma, autocontido, valorizando o grau de fidelidade aos founding fathers, outra parte busca uma postura diferente, de avanço substantivo na construção teórica, proposições relevantes para questões contemporâneas e maior diálogo e intercâmbio de ideias com o restante da profissão, sem abandonar a herança mengeriana, misesiana, hayekiana e kirzneriana. ${ }^{11}$

\section{A Escola Austríaca se reduz a um capítulo da histó- ria do pensamento econômico?}

Kirzner (1987), escrevendo na segunda metade da década de 1980, afirma que, ao menos para parte da profissão, a Escola Austríaca é um capítu-

11 Como será visto na próxima seção, muito do trabalho contemporâneo em Economia Austríaca passa pela aproximação com outras correntes de pensamento econômico. Por exemplo, sobre a possibilidade de aproximação com a Constitutional Economics e a Public Choice, ver Lea e Martin (2014). O’Driscoll e Rizzo (1985) propuseram a aproximação do Austrianismo com o pós-keynesianismo de Paul Davidson. Sobre tentativas de intercâmbio entre a Escola Austríaca e a abordagem de Elinor Ostrom, ver Boettke e Snow (2014). Boettke (1989) propõe a aproximação dos Austríacos com a Escola Institucional Original, em particular a de caráter mais vebleniano. Sobre a proximidade entre o Austrianismo e a Economia Experimental, ver Oprea e Powell (2010). Ver também os capítulos de 70 a 86 do livro editado por Boettke (org., 1994). 
lo encerrado da história do pensamento econômico. Segundo essa visão, a Escola Austríaca teria deixado de existir a partir do início da década de 1930, tendo sido ou absorvida pelo mainstream, ou superada pela macroeconomia keynesiana que então surgia. Isso, porém, não estaria de acordo com um paradigma progressivo, que é como García-Brazales (2002) qualifica a Escola Austríaca.

Como constata Aimar (2009), pode-se dizer, em certo sentido, que a Escola Austríaca acaba por sofrer consequências negativas em virtude de possuir um rico passado. O renascimento do austrianismo a partir de meados da década de 1970 trouxe consigo uma grande reavivamento do interesse pelos clássicos da tradição. Nesse movimento, um grande esforço foi feito para que se enfatizasse a releitura de textos antigos, por vezes num esforço de exegese. Ademais, um traço que marca muitos dos textos escritos por Austríacos é o número relativamente elevado de referências e citações a Hayek, Mises e outros autores pretéritos.

Tais características podem ajudar a difundir entre a comunidade de economistas a percepção de que por recorrerem tanto a textos antigos, supostamente, pouco dialogam com o que tem sido feito na fronteira do conhecimento, e tendem a isolar os Austríacos das discussões contemporâneas consideradas relevantes em nível teórico, em um contexto em que prevalece o ideal de hard science e de superação positiva (Arida, 1984), associado à concepção whig vinculada muitas vezes ao modo de se fazer HPE. ${ }^{12}$

Nesse contexto, a presente seção argumenta que é falsa a caracterização da Escola Austríaca como apenas um capítulo de história do pensamento econômico ou como um grupo dedicado apenas a discussões sobre o passado da disciplina. Isso se torna particularmente interessante em um contexto intelectual em que ser um capítulo da HPE, ou mesmo fazer HPE, significa, na opinião de boa parte da profissão, ser incapaz de produzir conhecimento novo e relevante para o desenvolvimento contemporâneo da ciência econômica e para a compreensão do mundo atual. ${ }^{13}$

12 A concepção de que existe uma oposição entre história do pensamento econômico e teoria econômica, e que a primeira seria de pouca valia para a compreensão da realidade contemporânea, se comparada à segunda, é relativamente comum entre os economistas, mas, de um ponto de vista austríaco, não é verdadeira. Ver, a respeito, Boettke (2000, 2001, 2002b) e Boettke et al. (2014).

13 A discussão sobre o (pouco) papel geralmente atribuído à HPE pela maior parte da profissão, bem como suas causas e consequências, é longo dentro deste ramo da Economia. Ver, por exemplo, Cesarano (1983), Foley (2009), Kates (2013), Caldwell (2013), Cardoso (1995), Boettke (2000) e Boulding (1971). Lodewijks (2003) fala de como muito da pesquisa hetero- 
Um indicador interessante para se compreender como o austrianismo contemporâneo não se reduz à HPE como esta é normalmente compreendida pela maior parte da profissão surge ao se investigar a parcela dos artigos publicados no principal periódico dos Austríacos, a Review of Austrian Economics (RAE), ${ }^{14}$ designam-se com o código B do Journal of Economic Literature (JEL). Como se sabe, a categoria B é reservada para HPE, metodologia e abordagens heterodoxas. O código B00 é simplesmente "geral", os códigos que começam com B1 tratam da HPE até 1925, os que começam com B2 dizem respeito à HPE após 1925, os que se iniciam com B3 tratam de indivíduos, aqueles com B4 tratam de metodologia econômica, e finalmente os que começam com B5 são reservados para abordagens heterodoxas contemporâneas. Dessa maneira, os códigos B1 a B3 podem ser vistos como dedicados exclusivamente a HPE, o B4 a metodologia. Já o B5 não necessariamente é dedicado a HPE, mas pode ser compreendido como destinado a teoria econômica heterodoxa atual. ${ }^{15}$

Entre 2013 e 2015, a RAE publicou os volumes 26, 27 e 28, cada um deles com 4 números. Nesses 12 números, 120 artigos foram publicados, sendo 28 deles resenhas. Dos 92 restantes que não eram resenhas, 81 apresentam códigos JEL. Desses 81, apenas 4 apresentavam exclusivamente códigos JEL de B1 a B4, vale dizer, eram artigos que, na visão dos próprios autores, lidavam exclusivamente com HPE ou metodologia. Por outro lado, 77 apresentavam pelo menos um código JEL que não pertencente às classificações entre B1 e B4, ou seja, continham ao menos parcialmente uma discussão ou um objetivo não propriamente ligados à HPE ou à discussão metodológica, e, desses, 41 não continham nenhum código entre B1 e B4. Assim, ao levarmos em conta os artigos publicados entre 2013 e 2015 na

doxa contemporânea se abriga sob o rótulo de HPE, como a HPE é feita, em boa parte, como crítica da Economia Ortodoxa, bem como das reações a isso, em particular de pesquisadores em HPE.

14 Existem dois periódicos científicos principais dedicados à Escola Austríaca: a RAE, publicada trimestralmente pela Springer e que tem como editores-chefes Peter Boettke e Christopher Coyne, ambos professores da GMU, e o Quarterly Journal of Austrian Economics (OJAE), que tem como editor Joseph Salerno, um dos expoentes do Instituto von Mises, que publica a revista. A consideração sobre a maior importância da $R A E$ em relação ao QJAE pode ser inferida a partir do trabalho de Combes e Linnemer (2010). Nele, a RAE possui um fator de impacto CLh de 0,48, estando na posição 437, ao passo que o QJAE possui fator de impacto 0,31, ocupando a posição 638 no ranking de revistas de Economia. Por sua vez, Lee et al. (2010) propõe um ranking de 63 revistas heterodoxas em que a $R A E$ ocupa a posição 37, ao passo que o QJAE toma a posição 51.

15 Para outra aplicação da pesquisa em artigos através do código JEL B, ver Duarte e Giraud (2016). 
$R A E$ com código JEL, mais da metade deles, na visão dos próprios autores, não tinham HPE ou metodologia como um tema central.

Se, além das resenhas, retirarmos os artigos que foram publicados por terem sido apresentados em congressos ou simpósios, além das palestras de posse dos presidentes da SDAE, restam 41 artigos com código JEL que, possivelmente, passaram pelo processo usual de submissão e avaliação. Desses, apenas dois continham apenas códigos entre B1 e B4, vale dizer, na visão de seus autores lidavam exclusivamente com HPE ou metodologia. Dos outros 39, 30 não continham códigos entre B1 e B4, ou seja, na visão de seus autores não faziam discussão sobre HPE ou metodologia.

Este é um indicativo de que parte relevante dos artigos escritos e lidos por economistas que de algum modo se relacionam com o austrianismo não enxerga seu trabalho como sendo de HPE ou metodologia no sentido usual do termo - o que, por certo, não implica que os autores do passado não sejam valorizados pelos Austríacos, assim como a discussão metodológica, na medida em que suas contribuições não se restringem à HPE ou à metodologia, mas são intimamente relacionadas com a própria teoria econômica. Evidentemente, tal fato pode estar relacionado à forma borrada com que os Austríacos, em geral, traçam a fronteira entre teoria econômica e história do pensamento econômico, conforme mencionado anteriormente.

Ao mesmo tempo, pode-se utilizar do insight oferecido por Biddle (1998) ao analisar se uma suposta "falha reprodutiva" institucionalista seria capaz de explicar o declínio daquela Escola ao longo do século XX nos EUA. Ou seja, se uma ausência de reposição de quadros nas universidades (supostamente mais atraídos para outros campos, em particular o serviço público) teria reduzido a formação de novos institucionalistas e a exposição de suas ideias para novas gerações de economistas, o que teria tido papel central na perda de prestígio e espaço daquela Escola.

Propõe-se aqui se adaptar essa proposta de Biddle (1998) ao caso dos Austríacos por meio da investigação sobre o emprego dos alunos de doutorado formados pelo programa de Economia da Universidade George Mason. Mais especificamente, busca-se informação daqueles que foram orientados pelos professores que são conhecidos como Austríacos (Peter Boettke, Christopher Coyne, Lawrence White e Peter Leeson, embora existam outros que sejam influenciados por autores Austríacos, ainda que não sejam tão identificados com a Escola quanto os quatro relacionados). 
Segundo a página do departamento de Economia da Universidade (http://economics.gmu.edu/defenses?page=11\&past=true, visitado em 10/04/2016), entre fevereiro de 2009 e abril de 2015, 35 doutores em Economia se formaram na GMU sob orientação daqueles quatro professores. Uma pesquisa pela internet nas páginas destes recém-doutores e em páginas das universidades indica que apenas quatro deles, em agosto de 2015, não estavam trabalhando em universidades. Isso é um indício de que a Escola Austríaca contemporânea não esteja sofrendo da "falha reprodutiva" de que fala Biddle (1998) ao levantar uma hipótese para a queda do institucionalismo norte-americano, na medida em que professores que foram possivelmente expostos a uma formação Austríaca estejam atuando na formação de novos economistas. ${ }^{16}$

Em tal contexto, Boettke (2011) recomenda que os Austríacos, ao escreverem seus trabalhos, não fiquem preocupados em demonstrar "fidelidade" aos grandes nomes associados à tradição; nesse sentido, não é importante a quantidade de citação a trabalhos de Mises e Hayek, por exemplo, mas sim a construção de argumentos a partir da abordagem proposta por Mises e Hayek que sejam relevantes para a solução e compreensão de problemas contemporâneos. Essa, ele diz, era a postura que os próprios Mises e Hayek tiveram ao longo de sua trajetória. ${ }^{17}$

De fato, possivelmente como consequência dessa visão, o grupo de economistas Austríacos que Boettke (2010a) chama de terceira geração (após o "ressurgimento" da Escola em 1974) tem levado adiante o que Stein e Storr (2013) chamam de applied turn. De acordo com eles, "[t]hese "third generation" scholars are primarily engaged in efforts to understand a specific phenomenon or group of phenomena in the real world" (Stein e Storr, 2013, p. 33). Com isso, as discussões e críticas metodológicas têm cedido espaço, no austrianismo, a trabalhos aplicados a partir da teoria construída pelas gerações anteriores. Stein e Storr (2013) trazem uma lista de trabalhos que tem caracterizado a terceira geração que emergiu ao longo dos últimos 10 anos.

16 Cumpre notar que a hipótese de Biddle (1998) diz respeito a professores engajados no ensino de pós-graduação ao invés de apenas na graduação, distinção que é ignorada no presente trabalho.

17 "It is not faithfulness in citation practices to the masters, and certainly not the number of block quotes you can provide from them, that makes a paper a worthy contribution to "Austrian" economics. It is instead the quality of the argument that you make, and its relevance to solving a significant problem in the economic and/or policy world" (Boettke, 2011, p. 22). 
Já Aimar (2009) cita três campos de desenvolvimento teórico austríaco: a teoria da firma (como desdobramento da teoria da ação empresarial de Kirzner), a nova análise institucional comparativa (ao estudo institucional da transição do socialismo para o capitalismo nos países do leste europeu) e a Economia regional.

Rizzo (2009), por seu turno, faz um mapeamento mais completo sobre os trabalhos de economistas Austríacos desde o final dos anos 1980. Já em 1996 ele havia percebido que "Austrian economics has changed in the past ten years and that change has been positive. Austrians have now become among the most creative, innovative and least doctrinaire of economists" (Rizzo, 1996, p. xiii). Não cabe aqui replicar a (vasta) lista de trabalhos levantados por Rizzo, mas é interessante notar que ela abrange diferentes tópicos relacionados à construção teórica contemporânea inclusive fora do austrianismo, o que sugere a possibilidade de aumento de intercâmbio com outras correntes contemporâneas do pensamento econômico. A divisão de trabalhos recentes feita por Rizzo abrange os seguintes tópicos: macroeconomia e teoria monetária; atividade empresarial; processos de mercado e instituições econômicas; ordens espontâneas; Law and Economics.

\section{Considerações finais}

O artigo procurou colaborar no esclarecimento de alguns pontos referentes à Escola Austríaca. Isso parece especialmente importante em um momento em que muitos estudantes de Economia começam a buscar maior conhecimento sobre o austrianismo, uma Escola que até o presente momento não tem conseguido grande penetração na academia brasileira de Economia, seja na pesquisa, seja no ensino. Com vistas a isso, foi argumentado que a Escola Austríaca não é ortodoxa, já que rejeita um dos três pilares neoclássicos, qual seja, a ênfase em estados de equilíbrio, e o substitui pela análise do processo de mercado. $\mathrm{O}$ artigo também mostrou que a Escola Austríaca tem, ao menos em parte, buscado um maior engajamento dentro da profissão, ao invés de adotar a estratégia do isolamento. Por fim, o texto apresentou porque a Escola Austríaca não deve ser reduzida a um capítulo fechado da história do pensamento econômico (no sentido normalmente adotado na profissão), na medida em que tem procurado avançar nos debates teóricos contemporâneos e na utilização de sua abordagem singular para problemas aplicados. 


\section{Referências}

AIMAR, T. The curious destiny of a heterodoxy: The Austrian economic tradition. Review of Austrian Economics, v. 22, n. 3, p. 199-207, 2009.

ANGELI, E. A agenda de pesquisa heterodoxa da mainline economics. Economia e Sociedade, v. 23, n. 3, p. 731-756, 2014.

ARIDA, P. A História do Pensamento Econômico como Teoria e Retórica, 1984. In: GALA, P.; REGO, J. M. (orgs.). A História do Pensamento Econômico como Teoria e Retórica: Ensaios sobre Metodologia em Economia. São Paulo: Editora 34, 2003.

BARBIERI, F. Filosofia da ciência como ferramenta microeconômica. Nova Economia, v. 16, n. 3, p. 507-534, 2006.

BARBIERI, F. O Ressurgimento da Escola Austríaca e a Teoria de Processo de Mercado. Econômica, v. 10, n. 2, p. 215-235, 2008.

BARBIERI, F. História do Debate do Cálculo Econômico Socialista. São Paulo: Instituto Ludwig von Mises Brasil, 2013.

BIDDLE, J. Institutional Economics: A Case of Reproductive Failure? History of Political Economy, v. 30, Supplement, p. 108-133, 1998.

BLOCK, W. Rejoinder to David Prychitko on Austrian Dogmatism. Reason Papers, v. 34, n. 2, p. 151-166, 2012.

BOETTKE, P. Evolution and Economics: Austrians as Institutionalists. Research in the History of Economic Thought and Methodology, v. 6, p. 73-89, 1989.

BOETTKE, Peter. Introduction. In: BOETTKE, P. (org.). The Elgar Companion to Austrian Economics. Aldershot: Edward Elgar, 1994.

BOETTKE, P. Why Are There No Austrian Socialists? Ideology, Science and the Austrian School. Journal of the History of Economic Thought, v. 17, n. 1, p. 35-56, 1995.

BOETTKE, P. Why read the classics in Economics? Library of Economics and Liberty (Online), 2000. Disponível em: http://www.econlib.org/library/Features/feature2.html. Acesso em: 10/04/2016.

BOETTKE, P. F. A. Hayek as an intellectual historian of economics. In: MEDEMA, S.; SAMUELS, W. (orgs.). Historians of Economics and Economic Thought: The construction of disciplinary memory. London: Routledge, 2001.

BOETTKE, P. Information and Knowledge: Austrian Economics in Search of its Uniqueness. Review of Austrian Economics, v. 15, n. 4, p. 263-274, 2002a.

BOETTKE, P. The Use and Abuse of the History of Economic Thought within the Austrian School of Economics. History of Political Economy, v. 34, sup. 1, p. 337-360, 2002 b.

BOETTKE, P. Liberty vs. power in economic policy in the 20th and 21st centuries. Journal of Private Enterprise, v. 32, n. 2, p. 7-36, 2007.

BOETTKE, P. Back to the future: Austrian economics in the twenty-first century. In: BOETTKE, P. (org.). Handbook on Contemporary Austrian Economics. Cheltenham: Edward Elgar, 2010a.

BOETTKE, P. New Thinking for a New Decade, Coordination Problem, 2010b. Disponível em: 
http://www.coordinationproblem.org/2010/01/new-thinking-for-a-new-decade-1.html. Acesso em: 20/07/2016.

BOETTKE, P. Teaching Austrian Economics to Graduate Students. Journal of Economics and Finance Education, v. 10, n. 2, p. 19-30, 2011.

BOETTKE, P. The Methodology of Austrian Economics as a Sophisticated, Rather Than Naive, Philosophy of Economics. Journal of the History of Economic Thought, v. 37, n. 1, p. 79-85, 2015.

BOETTKE, P.; COYNE, C.; LEESON, P. Earw(h)ig: I can't hear you because your ideas are old. Cambridge Journal of Economics, v. 38, n. 3, p. 531-544, 2014.

BOETTKE, P.; COYNE, C.; NEWMAN, P. The History of a Tradition: Austrian Economics from 1871 to 2016. Research in the History of Economic Thought and Methodology, v. 34A, p. 199-243, 2016.

BOETTKE, P.; SNOW, N. Political economy and the science of association: A suggested reconstruction of public choice through the alliance of the Vienna, Virginia, and Bloomington schools of political economy. Review of Austrian Economics, v. 27, n. 1, p. 97-110, 2014.

BOULDING, K. After Samuelson, Who Needs Adam Smith? History of Political Economy, v. 3, n. 2, p. 225-237, 1971.

CALDWELL, B. The case for pluralism. In: DE MARCHI, Neil (org.). The Popperian Legacy in Economics: Papers Presented at a Symposium in Amsterdam, December 1985. Cambridge: Cambridge University Press, 1988.

CALDWELL, B. Of Positivism and the History of Economic Thought. Southern Economic Journal, v. 79, n. 4, p. 753-767, 2013.

CARDOSO, J. L. Teaching the history of economic thought. The European Journal of the History of Economic Thought, v. 2, n. 1, p. 197-214, 1995.

CESARANO, F. On the role of the history of economic analysis. History of Political Economy, v. 15 , n. 1, p. 63-82, 1983.

COMBES, P.-P.; LINNEMER, L. Inferring Missing Citations: A Quantitative Multi-Criteria Ranking of all Journals in Economics. Groupement de Recherche en Economie Quantitative d'Aix-Marseille, Document de Travail n 2010-28, 2010. Disponível em: https://hal.inria. fr/halshs-00520325/document. Acesso em: 10/04/2016.

DEQUECH, D. Neoclassical, mainstream, orthodox, and heterodox economics. Journal of Post Keynesian Economics, v. 30, n. 2, p. 279-302, 2007.

DUARTE, P. G.; GIRAUD, Y. The Place of the History of Economic Thought in Mainstream Economics, 1991-2011, Viewed Through a Bibliographic Survey. Journal of the History of Economic Thought, v. 38, n. 4, p. 431-462, 2016.

EGGER, J. The Austrian Method, 1976. In: SPADARO, Louis (org.). New Directions in Austrian Economics. Kansas City: Sheed Andrews and McMeel, 1978.

FOLEY, D. The History of Economic Thought and the Political Economic Education of Duncan Foley. Journal of the History of Economic Thought, v. 31, n. 1, p. 21-31, 2009.

GARCÍA-BRAZALES, Á. R. Austrian economics as a progressive paradigm: explaining more complex economic phenomena. Review of Austrian Economics, v. 15, n. 4, p. 335-357, 2002. 
HAYEK, F. von. Economics and Knowledge, 1937. In: HAYEK, F. Individualism and Economic Order. Chicago: The University of Chicago Press, 1948.

HAYEK, F. von. The Use of Knowledge in Society, 1945. In: HAYEK, Friedrich von. Individualism and Economic Order. Chicago: The University of Chicago Press, 1948.

HAYEK, F. The Counter-Revolution of Science. Indianapolis: Liberty Fund, 1979.

KATES, S. Defending the History of Economic Thought. Cheltenham: Edward Elgar, 2013.

KIRZNER, I. Equilibrium versus Market Process, 1974. In: KIRZNER, I. Perception, Opportunity, and Profit. Chicago: The University of Chicago Press, 1983.

KIRZNER, I. The Austrian School of Economics, 1987. In: KIRZNER, I. The meaning of market process: essays in the development of modern Austrian economics. London: Routledge, 1992.

KIRZNER, I. The Kirznerian Way: An Interview with Israel M. Kirzner. Austrian Economics Newsletter, v. 17, n. 1, 1997. Disponível em: https://mises.org/library/kirznerian-wayinterview-israel-m-kirzner. Acessado em: 10/04/2016.

KIRZNER, I. Competition and Entrepreneurship. Indianapolis: Liberty Fund, 2013.

KLEIN, P. Foreword. In: MENGER, C. Principles of Economics. Auburn: Ludwig von Mises Institute, 2006.

KLEIN, P. The Mundane Economics of the Austrian School. The Quarterly Journal of Austrian Economics, v. 11, n. 3, p. 165-187, 2008.

KOPPL, R. Austrian economics at the cutting edge. Review of Austrian Economics, v. 19, n. 4, p. 231-241, 2006.

LACHMANN, L. An Austrian Stocktaking: Unsettled Questions and Tentative Answers. In: SPADARO, Louis (org.). New Directions in Austrian Economics. Kansas City: Sheed Andrews and McMeel, 1978.

LEA, G. S.; MARTIN, A. From Vienna to Virginia: Exchange, rules, and social cooperation. Review of Austrian Economics, v. 27, n. 1, p. 1-9, 2014.

LEE, F.; CRONIN, B.; McCONNELL, S.; DEAN, E. Research Quality Rankings of Heterodox Economic Journals in a Contested Discipline. American Journal of Economics and Sociology, v. 69, n. 5, p. 1409-1452, 2010.

LODEWIJKS, J. Research in the History of Economic Thought as a Vehicle for the Defense and Criticism of Orthodox Economics. In: SAMUELS, W.; BIDDLE, J.; DAVIS, J. (orgs.). A Companion to the History of Economic Thought. Malden: Blackwell, 2003.

MAIR, D.; MILLER, A. (orgs.). A Modern Guide to Economic Thought: An Introduction to Comparative Schools of Thought in Economics. Aldershot: Edward Elgar, 1991.

MISES, L. von. The Ultimate Foundation of Economic Science: An Essay on Method. Indianapolis: Liberty Fund, 2006.

MISES, L. von. Human Action: A Treatise on Economics. Indianapolis: Liberty Fund, 2007.

OPREA, R.; POWELL, B. Why Austrians should quit worrying and learn to love the lab. In: KOPPL, R.; HORWITZ, S.; DESROCHERS, P. (orgs.). What is so Austrian about Austrian Economics? (Advances in Austrian Economics, Volume 14). Bradford: Emerald, 2010. 
POSSAS, M. L. A cheia do mainstream: comentários sobre os rumos da ciência econômica. Revista de Economia Contemporânea, v. 1, n. 1, p. 13-58, 1997.

PRYCHITKO, D. L. Thoughts on Austrian Economics, 'Austro-Punkism' and Libertarianism. In: PRYCHITKO, D. L. Markets, Planning, and Democracy: Essays After the Collapse of Communism. Cheltenham: Edward Elgar, 2002.

RIZZO, M. J. Introduction: Time and Ignorance After Ten Years. In: O'DRISCOLL, Gerald; RIZZO, Mario. The Economics of Time and Ignorance, London, Routledge, 1996.

RIZZO, M. J. Austrian Economics: Recent Work. In: DURLAUF, S. N.; BLUME, L. E. (orgs.). The New Palgrave Dictionary of Economics (Online Edition). Palgrave: Macmillan, 2009.

RIZZO, M. Foundations of The Economics of Time and Ignorance. Review of Austrian Economics, v. 26, n.1, p. 45-52, 2013.

ROTHBARD, M. The Present State of Austrian Economics, 1992. In: ROTHBARD, M. Economic Controversies. Auburn: Ludwig von Mises Institute, 2011.

STEIN, S.; STORR, V. H. The difficulty of applying the economics of time and ignorance. Review of Austrian Economics, v. 26, n. 1, p. 27-37, 2013.

VAUGHN, K. Austrian Economics in America: The Migration of a Tradition. Cambridge: Cambridge University Press, 1994.

VAUGHN, K. Remembering Jim Buchanan. Review of Austrian Economics, v. 27, n. 2, p. 157164, 2014.

\section{Sobre o autor}

Eduardo Angeli - eduardoangeli@hotmail.com

Universidade Federal do Paraná, Curitiba, Paraná.

\section{Sobre 0 artigo}

Recebido em 26 de abril de 2016. Aprovado em 09 de janeiro de 2017. 